\title{
Calibração de esfigmomanômetros: levantamento a respeito do conhecimento de estudantes de medicina
}

\section{Calibration of sphygmomanometers: survey on the knowledge of medical students}

Frederico Antonio Rabelo ${ }^{\dagger^{*}}$, Tadeu Florido José Gama ${ }^{\dagger}$, Caio Teixeira dos Santos ${ }^{\dagger}$, Raul Ferreira de Souza Machado ${ }^{\dagger}$, Lívia Maria Horta Rodrigues ${ }^{\dagger}$, Marise Maleck ${ }^{\dagger, \$ \S}$

Como citar esse artigo. Rabelo, F.A.; Gama T.F.J.; Dos Santos, C.T.; Machado, R.F.S.; Rodrigues, L.M.H.; Maleck, M. Calibração de Esfigmomanômetros: um levantamento a respeito do conhecimento de estudantes de medicina. Revista de Saúde. 2020 Jul./Dez.; 11 (2): 15 - 19.

\section{Resumo}

A hipertensão arterial sistêmica (HAS) é uma condição clínica multifatorial caracterizada por elevação sustentada da pressão arterial (PA). A aferição correta da medida da PA é assegurada pelas condições adequadas de funcionamento do esfigmomanômetro e pelo uso da técnica correta. O trabalho teve como objetivo avaliar as condições de uso dos aparelhos de medida da PA e mostrar o nível de conhecimento técnico a respeito do assunto entre acadêmicos de Medicina de uma universidade privada do estado do Rio de Janeiro. Realizou-se um estudo observacional-transversal de prevalência através de questionário online, entrevistando 432 estudantes do $1^{\circ}$ ao $12^{\circ}$ período do Curso de Medicina, que responderam a 9 perguntas de múltipla escolha sobre condições de uso e manutenção dos esfigmomanômetros. Sobre o ano em que adquiriam o aparelho, 416 alunos (96,2\%) responderam se lembrar do ano de compra; sobre a manutenção do aparelho, 389 alunos (90\%) responderam que, desde que o adquiriram, este não foi calibrado. Questionados sobre orientações quanto à necessidade de calibração, 264 estudantes $(61,1 \%)$ disseram nunca ter sido orientados, enquanto $168(38,9 \%)$ disseram já ter recebido essa orientação. No que tange aos possíveis impactos da não calibração, 181 $(41,9 \%)$ afirmaram conhecimento e $251(58,1 \%)$ disseram não saber dos impactos relacionados ao uso de aparelhos descalibrados. Constatou-se a necessidade de que sejam inseridos, nos ambientes estudantis e hospitalares, alertas e estratégias para manutenção periódica dos esfigmomanômetros, a fim de que os critérios estabelecidos pelo Instituto Nacional de Metrologia, Qualidade e Tecnologia sejam cumpridos.

Palavras-chave: Hipertensão, Monitores de Pressão Arterial, Manutenção de Equipamento, Calibragem.

Systemic arterial hypertension (SAH) is a multifactorial clinical condition characterized by a sustained rise in blood pressure (BP). The correct measurement of the BP is insured by the proper operating conditions of the sphygmomanometer and using the correct technique. The purpose of this work is to evaluate the conditions of use of the BP measuring devices and to show the level of technical knowledge on this subject between academics of a private university in the state of Rio de Janeiro. It was conducted an observational cross-sectional study to estimate the prevalence of knowledge concerning the use of this instrument with an online survey interviewing 432 students from the first to the last year of medical school who answered to 9 questions about the conditions of use and maintenance of the devices. To the question about the year in which the device was purchased, 416 students $(96.2 \%)$ replied to remember the year of purchase; on the maintenance of the device, 389 students $(90 \%)$ replied that it has not been calibrated since they were purchased. When asked about receiving guidance on the need for calibration, 264 students $(61.1 \%)$ said they were never guided on this topic, while $168(38.9 \%)$ said they had already received this guidance. Regarding the possible impacts of non-calibration of the device, $181(41.9 \%)$ reported to have knowledge about it and $251(58.1 \%)$ said they were unaware of the effects related to using unbalanced devices. These reported outcomes confirm the need for a constant alert and strategies for these sphygmomanometers' long-term maintenance to be incorporated in the academic and hospital environments in order to meet all criteria of specific use purposes by National Institute of Metrology, Quality and Technology.

Keywords: Hypertension, Blood Pressure Monitors, Equipment Maintenance, Calibration.

\section{Introdução}

A hipertensão arterial sistêmica (HAS) é uma condição clínica multifatorial caracterizada por uma elevação sustentada da pressão arterial (PA). Além de possuir manifestações clínicas próprias, a HAS é, também, considerada um importante fator de risco para eventos cardiovasculares que correspondem à maior causa de mortes no Brasil e no mundo․ Segundo a $7^{\mathrm{a}}$ Diretriz Brasileira de Hipertensão Arterial, o diagnóstico baseia-se na exatidão da medida da pressão arterial ${ }^{2}$.

Existem diferentes tipos de aparelhos utilizados para fins de aferição da PA, sendo eles divididos entre

\footnotetext{
Afiliação dos autores: 1

†Curso de Medicina, Pró - Reitoria de Ciências Médicas, Universidade de Vassouras, Vassouras, RJ, Brasil

*Laboratório de Insetos Vetores e Mestrado Profissional em Ciências Ambientais, Universidade de Vassouras, Vassouras, RJ, Brasil.

${ }^{\S}$ Laboratório de Entomologia Médica e Forense, IOC, FIOCRUZ, Rio de Janeiro, RJ, Brasil.
} 
esfigmomanômetros manuais (de mercúrio, aneroide e eletrônico), capazes de fornecer a PA através do método auscultatório, e esfigmomanômetros automáticos (eletrônico), os quais utilizam os métodos oscilométrico e misto ${ }^{3}$. Um estudo de revisão de literatura publicado nos Anais do I Congresso Norte-Nordeste de Tecnologias em Saúde, no ano de 2018, após análise de 23 artigos científicos foi mostrada a existência de divergências nas medidas da PA feitas pelo esfigmomanômetro de mercúrio e o digital, encontrando superioridade do método auscultatório em detrimento do oscilométrico ${ }^{4}$.

A aferição correta da medida da PA é assegurada pelas condições adequadas de funcionamento do esfigmomanômetro e pelo emprego da técnica correta. No Brasil, a Portaria $n^{\circ} 24 / 96$ do Instituto Nacional de Metrologia, Qualidade e Tecnologia (INMETRO) determinou as condições necessárias para os esfigmomanômetros mecânicos, de medição nãoinvasiva, destinados a medir PA, ou seja, a conservação dos componentes do aparelho (pêra, borracha, velcro ou pinos, manômetro, coluna de mercúrio, válvula) e sua calibração ${ }^{5}$. Possíveis vazamentos na válvula, por exemplo, promovem a desinflação da bolsa, podendo ocasionar uma diminuição na PA sistólica e elevação da diastólica ${ }^{6}$.

$\mathrm{Na}$ prática médica, várias decisões com relação a diagnóstico, prognóstico e terapia são tomadas com base na aferição dos níveis pressóricos ${ }^{7}$. Todavia, a dificuldade de detecção da elevação da PA em um paciente pode o expor a complicações agudas e/ou crônicas, tais como acidente vascular encefálico (AVE), insuficiência cardíaca, insuficiência renal, retinopatia hipertensiva, aterosclerose, dentre outras ${ }^{8}$. Entre as doenças cardiovasculares (DCVs), a HAS correlacionase a $40 \%$ das mortes por AVE e $25 \%$ das mortes por doenças coronarianas (DC) no país ${ }^{9}$. Pressupõe-se que cerca de $3 \%$ de todos os atendimentos em salas de emergências decorram de elevações da $\mathrm{PA}^{10}$. Além disso, ela representa um custo social de grande relevância, uma vez que as DCVs são responsáveis por um grande número de internações ${ }^{2}$.

Considerada uma das doenças crônicas não trasmissíveis de maior prevalência, não é incomum que o diagnóstico de HAS ocorra tardiamente, o que acarreta maior risco de desenvolvimento de complicações ${ }^{11,12,13}$. Não obstante, mesmo nos grandes centros ainda é possível encontrar uma grande quantidade de irregularidades quanto a manutenção dos aparelhos. Um estudo trasnversal realizado na cidade de Belo Horizonte - MG, no ano de 2017, avaliou 337 esfigmomanômetros em serviços de atendimentos de hospitais públicos e privados e mostrou que 132 (39\%) deles estavam com a calibração vencida ${ }^{1}$.

Dessa forma, é importante que haja uma preocupação em manter os esfigmomanômetros em bom estado de funcionamento e dentro das normas estabelecidas pelo INMETRO $^{14}$ para obtenção de uma medida fidedigna da PA. Diversos estudos que avaliaram as condições desses aparelhos mostraram a precariedade de seu estado de conservação, bem como elevados índices de descalibração ${ }^{15}$. Isto mostra a relevância do presente estudo, cujos objetivos são avaliar as condições de uso dos aparelhos de medida da PA e evidenciar o nível de conhecimento técnico, a respeito do assunto entre acadêmicos de Medicina de uma universidade privada do estado do Rio de Janeiro.

\section{Material e Métodos}

Trata-se de um estudo observacional-transversal de prevalência. $\mathrm{O}$ critério de inclusão utilizado foi o participante estar matriculado no curso de Medicina da Universidade de Vassouras, Vassouras, RJ. O estudo foi aprovado pelo Comitê de Ética em Pesquisa (CEP) da Universidade, sob parecer $\mathrm{n}^{\circ} 3.569 .357$, de 11 de novembro de 2019.

A coleta das informações foi realizada através de um link, produzido pelo aplicativo Google Docs ${ }^{\circledR}$, contendo um questionário, preservando o sigilo e a identidade dos participantes, durante o mês de março de 2020. O questionário anônimo foi composto de nove perguntas, objetivas e de múltipla escolha, sendo elas: “(1) Qual período letivo você cursa atualmente?" com as opções de respostas $\left(1^{\circ}\right.$ período, $2^{\circ}$ período, $3^{\circ}$ período, $4^{\mathrm{o}}$ período, $5^{\mathrm{o}}$ período, $6^{\mathrm{o}}$ período, $7^{\circ}$ período, $8^{\mathrm{o}}$ período e Internato). "(2) Você se lembra do ano em que adquiriu o seu esfigmomanômetro?" com as opções de respostas (Sim e Não). "(3) Em que ano adquiriu o aparelho?" com opções de resposta (Não lembra o ano, 2015 ou antes, 2016, 2017, 2018, 2019 e 2020). “(4) Nesse intervalo, já calibrou o seu aparelho?" com as opções de respostas (Sim e Não). "(5) Quantas vezes calibrou o seu aparelho?" com as opções de respostas (Não calibrou, $1 \mathrm{x}, 2 \mathrm{x}, 3 \mathrm{x}, 4 \mathrm{x}$ ou mais). "(6) Você já foi orientado alguma vez sobre a necessidade de recalibrar?" com as opções de respostas (Sim e Não). "(7) Você sabe os impactos que um aparelho não calibrado pode causar?" com as opções de respostas (Sim e Não). "(8) Quantos dias por semana, aproximadamente, você utiliza o seu esfigmomanômetro?" com as opções de respostas $(0,1$, $2,3,4,5$ ou mais). "(9) você tem interesse que nossos pesquisadores calibrem o seu esfigmomanômetro?" com as opções de respostas (Sim e Não).

Este link foi disponibilizado via aplicativo de mensagem instantâneo WhatsApp ${ }^{\circledR}$ aos 1.182 participantes, alunos das turmas do $1^{\circ}$ ao $12^{\circ}$ período. Foi realizada análise de cálculo amostral na população - com grau de confiança de $99 \%$ e margem de erro de até $5 \%$ - obtendo-se como tamanho representativo da 
amostra 427 alunos participantes. Os dados obtidos pelo questionário foram transformados em gráficos, disponibilizados pelo próprio aplicativo Google Docs ${ }^{\circledR}$, e em planilha do Microsoft Office Excel 2007®. Realizou-se a ferramenta de tabela dinâmica para correlacionar variáveis e foi feita análise quantitativa das respostas nos questionários.

\section{Resultados}
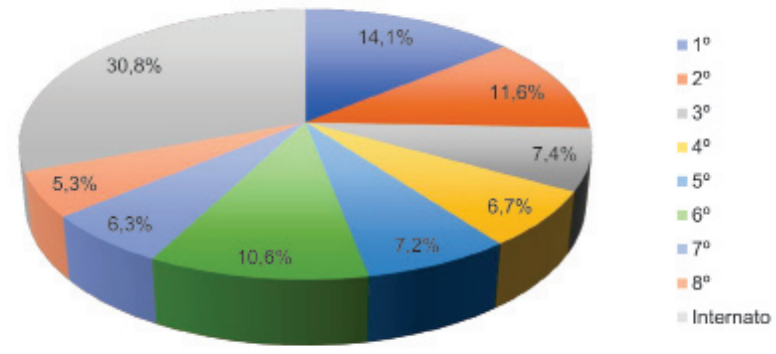

Figura 1. Porcentagem de alunos entrevistados em cada período da graduação.

Foram registradas respostas de 432 participantes, $61(14,1 \%)$ cursavam o primeiro período; $50(11,6 \%)$ o segundo período;32 (7,4\%) o terceiro período; $29(6,7 \%)$ o quarto período;31 (7,2\%) o quinto período; $46(10,6 \%)$ o sexto período;27 (6,3\%) o sétimo período; 23 (5,3\%) o oitavo período e 133 (30,8\%) o Internato (Figura 1). Conforme as Diretrizes Curriculares Nacionais do Curso de Graduação em Medicina, o Internato compreende os períodos letivos $9^{\circ}, 10^{\circ}, 11^{\circ}$ e $12^{\circ}$, durante os quais as atividades práticas estão sob a supervisão dos docentes da própria instituição ${ }^{16}$.

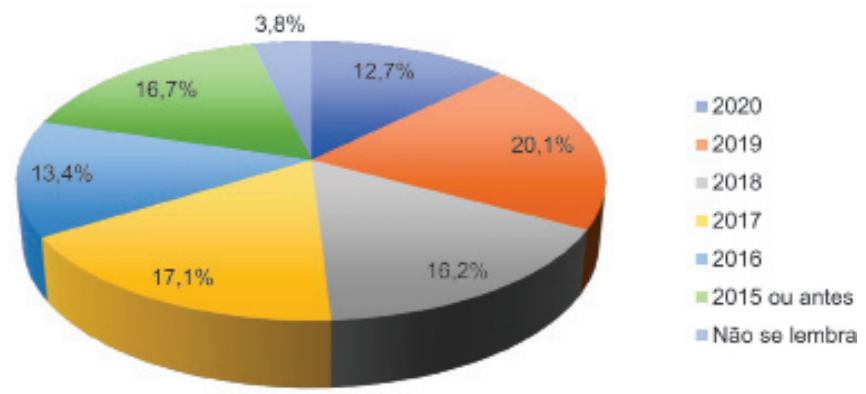

Figura 2. Porcentagem de alunos entrevistados que adquiriram seus aparelhos de esfigmomanômetro por ano.

Destes, 416 alunos $(96,2 \%)$ se lembravam do ano em que adquiriram o seu esfigmomanômetro, dos quais: $55(12,7 \%)$ no ano de $2020 ; 87(20,1 \%)$ em $2019 ; 70$
$(16,2 \%)$ em 2018; 74 (17,1\%) em 2017; $58(13,4 \%)$ em $2016 ; 72(16,7 \%)$ em 2015 ou antes e $16(3,8 \%)$ não se lembravam (Figura 2).

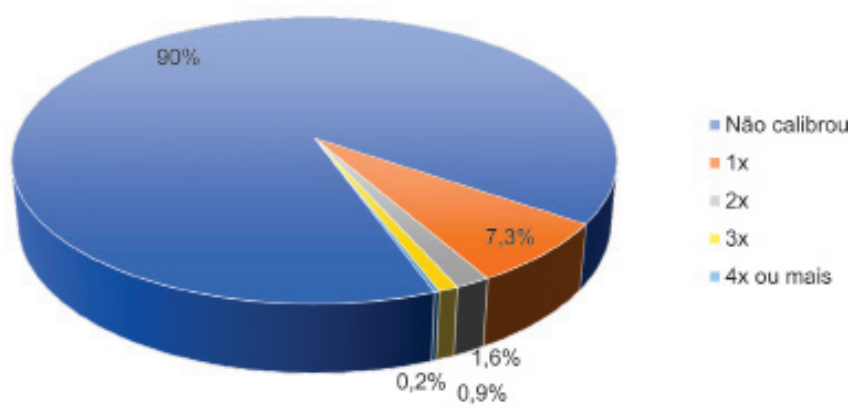

Figura 3. Porcentagem de alunos entrevistados que já calibraram seus aparelhos e número de calibrações realizadas.

Além disso, 389 alunos (90\%) responderam que, desde que adquiriram o aparelho, este não foi calibrado, enquanto $43(10 \%)$ responderam que já o calibraram. Em relação ao número de calibrações, 31 (7,3\%) relataram já ter calibrado o aparelho apenas uma vez; 7 $(1,6 \%)$ duas vezes; $4(0,9 \%)$ três vezes; $1(0,2 \%)$ quatro vezes ou mais. De acordo com a figura 3 , o total de 320 alunos (74\%) responderam que o esfigmomanômetro foi adquirido entre os anos de 2015 e 2019 do mesmo modo que, nesse intervalo, o aparelho não foi calibrado.

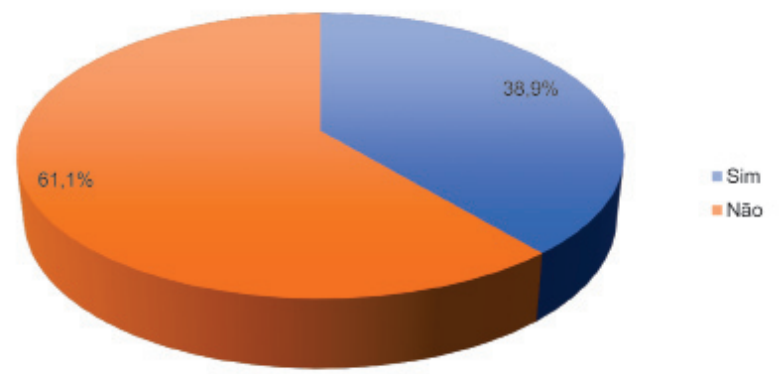

Figura 4. Porcentagem de alunos entrevistados que já foram orientados sobre a necessidade de recalibração de seus aparelhos.

Quando questionados sobre já terem recebido orientação quanto à necessidade de calibrar o esfigmomanômetro, 264 estudantes $(61,1 \%)$ disseram que nunca haviam sido orientados, enquanto $168(38,9 \%)$ disseram já ter recebido essa orientação (Figura 4). No que tange aos possíveis impactos da não calibração, $181(41,9 \%)$ afirmaram conhecimento e $251(58,1 \%)$ disseram não saber dos impactos relacionados ao uso de aparelhos descalibrados (Figura 5).

Os 207 alunos $(47,9 \%)$ afirmaram não ter sido orientados quanto aos riscos, além de não saberem as 


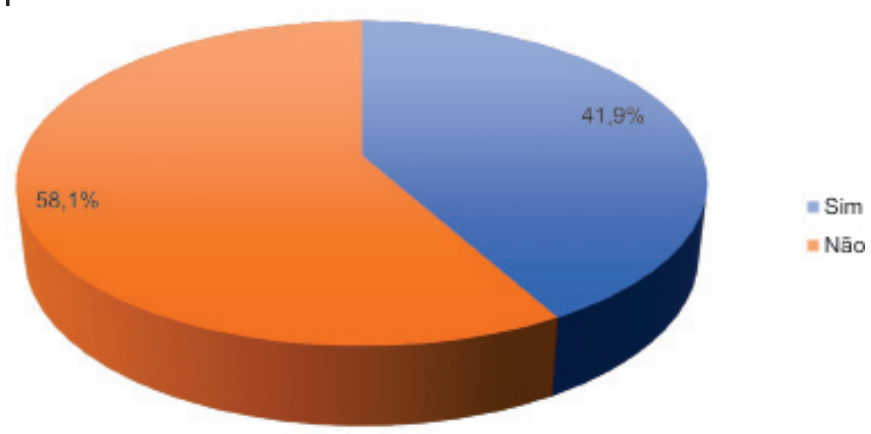

Figura 5. Porcentagem de alunos entrevistados que afirmam estar cientes acerca dos possíveis impactos da utilização de aparelhos não calibrados.

consequências a que são expostos os pacientes. Ainda, 158 alunos $(36,6 \%)$ alegaram a obtenção do aparelho há ao menos 01 ano e este não ter sido calibrado nos últimos seis meses, e que em nenhum momento lhes foi informado a respeito dessa necessidade, além de não saberem os impactos que essa falha técnica causaria.

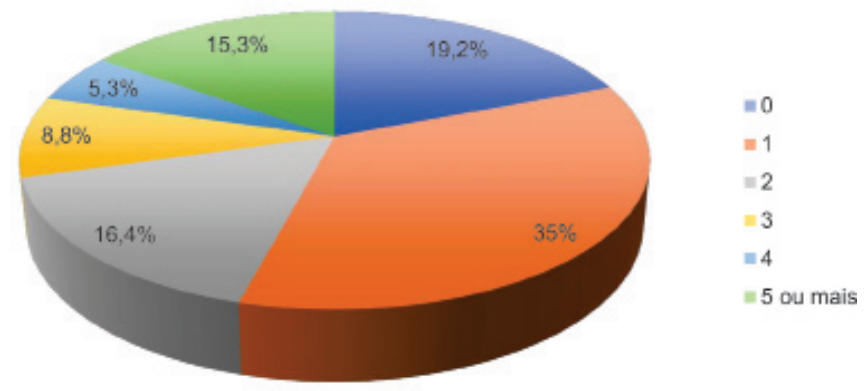

Figura 6. Porcentagem da frequência de uso dos aparelhos pelos alunos entrevistados.

Quanto à frequência de uso do aparelho, os entrevistados responderam: $83(19,2 \%)$ nenhum dia na semana;151 (35\%) uma vez na semana; $71(16,4 \%)$ duas vezes na semana; $38(8,8 \%)$ três vezes na semana; $23(5,3 \%)$ quatro vezes na semana e $66(15,3 \%)$ cinco

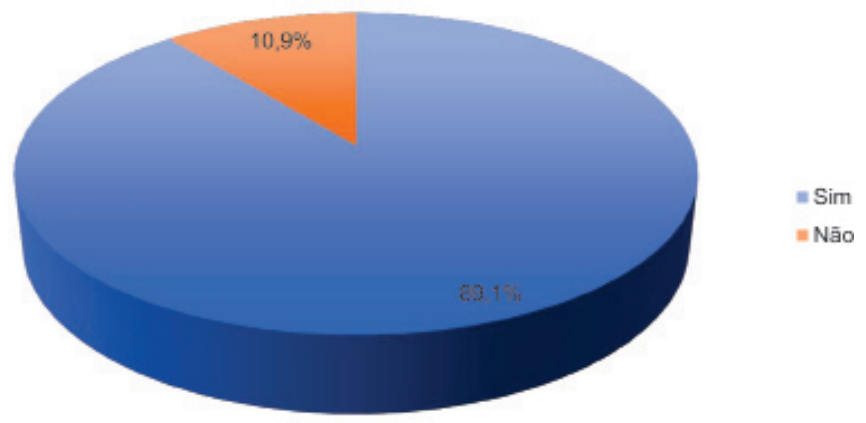

Figura 7. Porcentagem de alunos entrevistados que manifestaram interesse em ter seus aparelhos calibrados. vezes na semana ou mais (Figura 6).

Além disso, 385 alunos $(89,1 \%)$ manifestaram interesse em que os pesquisadores calibrassem seus aparelhos de esfigmomanômetro durante a pesquisa, enquanto $47(10,9 \%)$ não demonstraram interesse em ter seu aparelho calibrado (Figura 7).

\section{Discussão}

Sabe-se que o diagnóstico preliminar e preciso da HAS, a partir de aferição correta da pressão arterial, possibilita o diagnóstico precoce e a avaliação da eficácia do tratamento realizado, pautado não só na ação medicamentosa, mas também em mudanças educativas do estilo de vida ${ }^{6}$. A $7^{\circ}$ Diretriz Brasileira de Hipertensão Arterial recomenda a aferição da PA pelo menos a cada dois anos para adultos com $\mathrm{PA} \leq 120 / 80$ $\mathrm{mmHg}$, e anualmente para os indivíduos com PA entre $120 / 80 \mathrm{mmHg}$ e 140/90 mmHg. É importante salientar que os pacientes hipertensos podem apresentar hiato auscultatório, situação em que ocorre o desaparecimento temporário e precoce dos sons entre as fases I e II dos sons de Korotkoff, levando o observador a subestimar a pressão sistólica ou superestimar a diastólica ${ }^{2}$.

De acordo com os dados obtidos neste estudo, dos 416 alunos $(96,2 \%)$ que se lembravam do ano em que adquiriram seu esfigmonamômetro, 361 (83,5\%) $\mathrm{o}$ adquiriram há mais de um ano. Nesse universo, 320 alunos adquiriram o aparelho entre os anos de 2015 e 2019 e não o calibraram neste intervalo. Ademais, 158 $(36,6 \%)$ alunos afirmaram ter o aparelho há ao menos um ano e nunca o terem calibrado ou sido orientados a respeito, além de não saberem os possíveis impactos da utilização de aparelhos descalibrados.

Visto que se tratam de aparelhos em uso na comunidade local e nas instituições de saúde as quais os acadêmicos atuam semanalmente, os dados acima descritos mostram-se de grande relevância e expressividade, corroborando dados obtidos em estudo anterior, mostrando que $76,6 \%$ dos entrevistados não sabiam a frequência de calibragem de seus aparelhos, e quase a totalidade destes $(96,3 \%)$ não tinham data da última avaliação ${ }^{15}$.

Dentre os entrevistados do presente estudo, 389 alunos (90\%) afirmaram a não calibragem de seu aparelho manômetro aneróide no último ano, enquanto apenas 31 alunos $(7,3 \%)$ já o calibraram, porém apenas uma vez, contrariando a Portaria do INMETRO n 24 de 22 de fevereiro de 1996, que determina que estes aparelhos sejam calibrados a cada seis meses ${ }^{14}$.

Ressalta-se, ainda, dentre os 432 alunos, 264 $(61,1 \%)$ deles, relataram não terem sido orientados quanto à importância e a necessidade de realizar a calibragem semestral, e 251 alunos $(58,1 \%)$ não tinham conhecimento a respeito dos riscos e dos impactos que 
isso pode causar na prática e na tomada de decisão.

Estudos demonstraram que o uso de aparelhos descalibrados é responsável por $20 \%$ a $28 \%$ dos casos de HAS não diagnosticados e por $15 \%$ a $31 \%$ dos casos de hipertensão sistólica e diastólica falsamente diagnosticados, respectivamente. Vale ressaltar que obter medidas da PA falsamente elevadas pode ocasionar em erro diagnóstico e no uso indevido de medicamentos anti-hipertensivos desnecessariamente por um longo período da vida ${ }^{8}$. Dentre os possíveis desfechos causados pelo tratamento impróprio da HAS, relatam-se aumento do risco de lesões cardíacas, cerebrais e renais, bem como, alta frequência de internações e custos socioeconômicos elevados, levando a consequências diretas na produtividade e na renda familiar ${ }^{2}$.

Cabe destacar que 385 alunos $(89,1 \%)$ manifestaram interesse em ter os seus aparelhos calibrados ao final da pesquisa. Em contrapartida, 389 alunos (90\%) afirmaram que não realizaram a calibração do aparelho no último ano.

A abordagem do assunto na grade curricular ${ }^{16}$ dos cursos de Medicina e outros da área de saúde se expressa como estratégia viável e importante de difusão dessa informação, a fim de que maior número de futuros profissionais adquiram e possam difundir o hábito de manter seus esfigmonamômetros calibrados adequadamente, uma vez que 207 alunos (47,9\%) afirmaram não terem sido orientados sobre o risco, além de não saberem das consequências dessa falta de calibragem.

Além disso, é fundamental que haja uma maior atenção por parte das Instituições de Saúde para com a utilização e manutenção dos instrumentos utilizados, a fim de manter a aferição dentro dos parâmetros e normas estipulados pelo INMETRO.

\section{Conclusão}

A partir dos resultados obtidos, observou-se que, apesar do conhecimento sobre a correta utilização e conservação dos esfigmomanômetros, muitos estudantes não a realizam, de modo que muitos podem estar descalibrados e levando a interpretações errôneas, com resultados tanto falso-positivos quanto falso-negativos com relação ao diagnóstico da HAS. Evidencia-se, portanto, a necessidade de que sejam inseridos, nos ambientes estudantis e hospitalares, alertas e estratégias para a manutenção periódica desses aparelhos. Constatase, ainda, que ações coletivas para manutenção dos esfigmomanômetros devem ser estimuladas durante a grade curricular estudantil, respeitando-se, assim, os critérios técnicos de padronização para sua correta utilização.

\section{Referências}

1. Maia KAP, Malachias MVB, Paiva IV, Mariano FM, Paiva RV Inadequações dos esfigmomanômetros utilizados em serviços de urgência e emergência de uma grande capital brasileira. Int J Cardiovasc Sci2017 Dez; 30 (2): $100-8$.

2. Malachias MVB, Plavnik FL, Machado CA, Malta D, Scala LCN, Fuchs S, et al. $7^{\mathrm{a}}$ Diretriz brasileira de hipertensão arterial. Arq Bras Cardiol 2016 Set; 107(3): 1-6.

3. Comissão Setorial para a Saúde. A metrologia na saúde guia de boas práticas - parte II. Portugal: Instituto Português da Qualidade;2016.

4. Pierot EV, da Silveira FDR, Oliveira MCN, Amaral JV, Silva EP, Duarte GM. Utilização de aparelhos digitais para aferição de pressão arterial por profissionais de saúde. I Congresso Norte-Nordeste de Tecnologias Em Saúde [evento na internet]. 2018 Dez; Teresina, Brasil [acesso em 9 mai 2020]. Disponível em: https://revistas.ufpi.br/index.php/connts/article/ view/8095/4814

5. Lessa LBV, Garrido FSRG, Garrido RG. Manutenção e calibração de esfigmomanômetros: possíveis impactos na medição da pressão arterial. Colloq Vitae 2014Jun; 6 (3): 91-8.

6. Silva JB, Trubian FC, Lucacin AL, Rodrigues AM. Qualidade dos esfigmomanômetros aneroides utilizados por profissionais de saúde nas unidades do SUS em Cacoal - RO.RevElet FACIMEDIT 2016 Ago; 5 (2): $118-130$

7. Toledo MAV, Santos Neto LL, Santelo MN, Lima RSA, Arnaut LT. Validade dos esfigmomanômetros utilizados por profissionais de saúde do Hospital Universitário da Universidade de Brasília. Brasília Med 2002 Set; 39 (1/4): 5-9.

8. Tinoco MC. The accuracy of blood pressure measurement. Int J Cardiovasc Sci 2017 Abr; 30(2): 98-99.

9. Oliva MM, Biancolino CA. O impacto da inobservância da padronização da medida da pressão arterial segundo a AHA como evento adverso para o diagnóstico e tratamento da hipertensão arterial. Anais do III Simpósio Internacional de Gestão de Projetos, Inovação e Sustentabilidade - SINGEP, 2014. São Paulo, Brasil.

10. Marik PE, Varon J. Hypertensive crises: challenges and management. Chest 2007 Nov;131(6):1949-62.

11. Santos TC, Souza MTM, Mendonça NA, Beltrão CAMS, Sousa JDB, Barbosa TL, et al. Qualidade de vida em idosos hipertensos e diabéticos acompanhados pela estratégia saúde da família no município de Manaus, Amazonas. Congresso brasileiro de ciências sociais e humanas em saúde [evento na internet] 2019 João Pessoa, Brasil [acesso em 9 mai 2020]. Disponível em: https://proceedings.science/saude-coletiva-2018/papers/ qualidade-de-vida-em-idosos-hipertensos-e-diabeticos-acompanhados-pelaestrategia-saude-da-familia-no-municipio-de-manau.

12. Pinto BCM, Souza FPG, Costa IVL, Toledo LA, Souza TAF, Dias VCA et al. Hipertensão e diabetes mellitus: rastreamento e conscientização em uma indústria de Betim/MG. Sinapse Múltipla 2017 Dez; 6(2): 278-283.

13. Carvalho MR, Pires CML, Shuengue CMLO. Rastreamento da hipertensão e diabetes mellitus em funcionários de uma instituição em Manhuaçu-MG. Anais do IV Seminário Científico do UNIFACIG [evento na internet] 2018 Nov Manhuaçu, Brasil [acesso em 9 mai 2020]. Disponível em: http:// pensaracademico.facig.edu.br/index.php/semiariocientifico/article/view/777

14. Ministério da Indústria, do Comércio e do Turismo (Brasil). Portaria $n^{\circ} 24$, de 22 de fevereiro de 1996. Regulamentada no que se refere às competências do Instituto Nacional de Metrologia, Normalização e Qualidade Industrial INMETRO. Dispõe sobre a necessária regulamentação dos instrumentos de medir na área de saúde.

15. de Souza ST, de Andrade TG, de Gusmão JL, Colósimo FC, da Silva SSBE, Pierin AMG. Avaliação das condições de uso de esfigmomanômetros em serviços hospitalares. Acta Paul Enferm 2012 Mai; 25(6): 940-946.

16. Diretrizes curriculares nacionais do curso de graduação em medicina [Internet]. Brasil: Ministério da Educação; 2018. diretrizes curriculares nacionais; [cited 2020 May 27]; Available from: http://portal.mec.gov.br/ cne/arquivos/pdf/Med.pdf. 